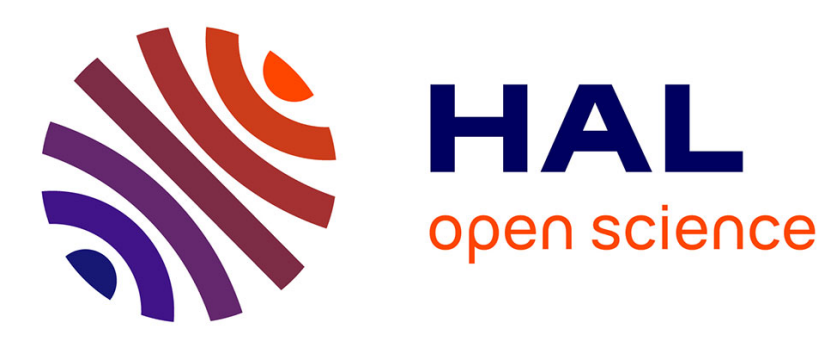

\title{
The hard sphere quantum propagator: exact results via partial wave analysis
}

Eugène de Prunelé

\section{To cite this version:}

Eugène de Prunelé. The hard sphere quantum propagator: exact results via partial wave analysis. Journal of Physics A: Mathematical and Theoretical, 2008, 41 (25), 10.1088/1751$8113 / 41 / 25 / 255305$. hal-00288744

\section{HAL Id: hal-00288744 \\ https://hal.science/hal-00288744}

Submitted on 22 Aug 2018

HAL is a multi-disciplinary open access archive for the deposit and dissemination of scientific research documents, whether they are published or not. The documents may come from teaching and research institutions in France or abroad, or from public or private research centers.
L'archive ouverte pluridisciplinaire HAL, est destinée au dépôt et à la diffusion de documents scientifiques de niveau recherche, publiés ou non, émanant des établissements d'enseignement et de recherche français ou étrangers, des laboratoires publics ou privés. 


\title{
The hard sphere quantum propagator: exact results via partial wave analysis
}

\author{
E de Prunelé \\ Institut UTINAM, UMR CNRS 6213, Université de Franche-Comté, 16 route de Gray, \\ 25030 Besançon Cedex, France
}

\begin{abstract}
An exact expression for the partial wave hard sphere quantum propagator in threedimensional space is given in terms of the Fourier transform of an expression involving spherical Bessel functions. For the $\mathrm{s}$ wave case, the Fourier transform is calculated analytically. This Fourier transform is also evaluated for the general case by contour integration in terms of a Laplace transform and residues contributions. The accuracy of previous approximations for a hard sphere quantum propagator can thus be evaluated. In particular, the Van Vleck-Gutzwiller classical approximation is found to be very accurate provided that the classical action is greater than only a few units of reduced Planck constant.
\end{abstract}

\section{Introduction}

Propagators are important conceptual tools in quantum mechanics. For a given physical system, propagators are matrix elements of the time evolution operator $U\left(t_{f}, t_{i}\right)$ which can be used to obtain the state $\left|\psi\left(t_{f}\right)\right\rangle$ of the system at time $t_{f}$ if its state at time $t_{i}$ is $\left|\psi\left(t_{i}\right)\right\rangle$. In the Schrödinger picture : $\left|\psi\left(t_{f}\right)\right\rangle=U\left(t_{f}, t_{i}\right)\left|\psi\left(t_{i}\right)\right\rangle$. For example, they are studied and discussed in the non-relativistic context in [1] and in both non-relativistic and relativistic contexts in [3]. Propagators are particularly important for Feynman formulation of quantum mechanics in terms of path integrals.

In the non-relativistic context to be considered here, analytic exact expressions for propagators are known for only a few simple systems, for example, free particles or harmonic oscillators [1,2]. An exact analytic expression for the propagator of a particle interacting only with a hard sphere is not known. This has motivated the search for approximate expressions. The so-called image approximation propagators [4-6] have been the first ones. They have now been superseded by a more recent attempt of Cao and Berne [7], to be called hereafter the CB propagator or the $\mathrm{CB}$ approximation. The hard sphere propagator, for real or imaginary time, 
is a basic ingredient for the study of thermodynamic properties of hard sphere systems (fluid or solid) and Bose-Einstein condensation of gases [8-16]. The CB approximation is based on a purely quantal approach to scattering, and has largely been used in these applications. But there is also another approximation for the hard sphere quantum propagator [17-19], involving only classical quantities as inputs. We shall call it the Van Vleck-Gutzwiller propagator, to be called hereafter the VG propagator or the VG approximation, which has been used for example in [20].

In the present paper, an exact expression for the partial wave hard sphere quantum propagator is given in terms of the Fourier transform of an expression involving spherical Bessel functions. This Fourier transform is also evaluated by contour integration in terms of a Laplace transform and residues contribution (see section 4.3). The accuracy of previous attempts at approximations for the hard sphere quantum propagator can thus be evaluated by comparison with exact results obtained by numerical integration of the exact expressions based on Fourier or Laplace transforms. It is also shown that for the s wave hard sphere quantum propagator, the Fourier transform can be calculated analytically, leading to a simple expression (see equation (15)).

Our starting point is the well-known expression (see, e.g., $[3,21]$ ) of the retarded time evolution operator as a Fourier transform of the resolvent $G(z)=(z-H)^{-1}$ for a system with time-independent Hamiltonian:

$$
\theta\left(t_{f}-t_{i}\right) U\left(t_{f}-t_{i}\right)=-\frac{1}{2 \pi \mathrm{i}} \int_{-\infty}^{+\infty} \mathrm{d} E \exp \left(-\mathrm{i} \frac{E\left(t_{f}-t_{i}\right)}{\hbar}\right) G\left(E_{+}\right) .
$$

The Heaviside function $\theta$ is zero when its argument is negative, and unity when its argument is positive. The $z$-plane of $G(z)$ is cut along the real positive axis, and the notation $G\left(E_{+}\right)$means $\lim _{\varepsilon->0} G(E+\mathrm{i} \varepsilon)$ with $\varepsilon>0$. Otherwise stated, on the integration interval $[0,+\infty]$, the cut has to be approached from above, i.e. with a vanishing positive imaginary part. Equation (1) can be verified by considering the inverse Fourier transform [21].

From now on, only the positive time interval $t=t_{f}-t_{i}$ will be considered, so that the Heaviside function will not appear in all the following.

\section{An exact expression in terms of Fourier transform}

What is the expression of the resolvent for a particle of mass $M$, in the three-dimensional space, interacting only with a hard sphere of radius $a$ ? To answer this question, let us first consider the Hamiltonian $H(\lambda)$ :

$$
\begin{aligned}
& H(\lambda)=H_{0}+\lambda V \\
& H_{0}=\frac{p^{2}}{2 M} \\
& V=a^{3} \sum_{\ell=0}^{+\infty} \sum_{m=-\ell}^{\ell}|a, \ell, m\rangle\langle a, \ell, m| .
\end{aligned}
$$

The vector $|r, \ell, m\rangle$ is a eigenvector of the squared orbital angular momentum with eigenvalue $\ell(\ell+1) \hbar^{2}$, an eigenvector of the component $\ell_{z}$ of the orbital angular momentum with eigenvalue $m \hbar$, and a generalized eigenvector of the radial position operator with generalized eigenvalue $r$. All operators are relative to a given frame $O, x, y, z$. The normalization is [22]

$$
\left\langle r^{\prime}, \ell^{\prime}, m^{\prime} \mid r, \ell, m\right\rangle=\frac{\delta\left(r^{\prime}-r\right)}{r^{2}} \delta_{\ell \ell^{\prime}} \delta_{m m^{\prime}}
$$


It has been shown (see equation (13) of [22]) that the stationary scattering partial wave functions for $H(\lambda)$ are

$\langle r, \ell, m \mid p, \ell, m,+\rangle=\mathrm{i}^{\ell} \sqrt{\frac{2}{\pi}}\left\{j_{\ell}(p r)-\frac{j_{\ell}(p a)}{\frac{1}{\lambda 2 M a^{3}}+p h_{\ell}^{+}(p a) j_{\ell}(p a)} p h_{\ell}^{+}(p r) j_{\ell}(p a)\right\}$

with $r \geqslant a$. It is easy to verify that for the limiting case $\lambda \rightarrow+\infty$, these stationary scattering partial wavefunctions are eigenfunctions of $H_{0}$ with vanishing boundary condition on the sphere of radius $a$ (see also [23]). It can also be noted that an explicit expression for the partial wave-scattering amplitude for $H(\lambda)$ can be obtained (see equation (12) of [22]). For the limiting case $\lambda \rightarrow+\infty$, these partial wave-scattering amplitudes then lead exactly to the phase shift for hard sphere scattering (compare with equation (50) of section $X-13$ of [24]).

Now we are interested in the partial-wave hard sphere propagators:

$\left\langle r_{2}, \ell, m|U(t)| r_{1}, \ell, m\right\rangle=-\frac{1}{2 \pi \mathrm{i}} \int_{-\infty}^{\infty} \mathrm{d} E \exp \left(-\mathrm{i} \frac{E t}{\hbar}\right)\left\langle r_{2}, \ell, m\left|G\left(E_{+}\right)\right| r_{1}, \ell, m\right\rangle$

and we first consider a partial-wave resolvent for the Hamiltonian $H(\lambda)$. From the algebraic identity

$$
G_{\lambda}(z)=G_{0}(z)+G_{0}(z) \lambda V G_{\lambda}(z)
$$

one obtains

$$
\begin{gathered}
\left\langle r_{2}, \ell, m\left|G_{\lambda}(z)\right| r_{1}, \ell, m\right\rangle=\left\langle r_{2}, \ell, m\left|G_{0}(z)\right| r_{1}, \ell, m\right\rangle \\
\quad+\lambda a^{3}\left\langle r_{2}, \ell, m\left|G_{0}(z)\right| a, \ell, m\right\rangle\left\langle a, \ell, m\left|G_{\lambda}(z)\right| r_{1}, \ell, m\right\rangle \\
\left\langle a, \ell, m\left|G_{\lambda}(z)\right| r_{1}, \ell, m\right\rangle=\left\langle a, \ell, m\left|G_{0}(z)\right| r_{1}, \ell, m\right\rangle \\
+\lambda a^{3}\left\langle a, \ell, m\left|G_{0}(z)\right| a, \ell, m\right\rangle\left\langle a, \ell, m\left|G_{\lambda}(z)\right| r_{1}, \ell, m\right\rangle \\
\left\langle a, \ell, m\left|G_{\lambda}(z)\right| r_{1}, \ell, m\right\rangle=\frac{\left\langle a, \ell, m\left|G_{0}(z)\right| r_{1}, \ell, m\right\rangle}{1-\lambda a^{3}\left\langle a, \ell, m\left|G_{0}(z)\right| a, \ell, m\right\rangle}
\end{gathered}
$$

so that finally

$$
\begin{aligned}
& \left\langle r_{2}, \ell, m\left|G_{\lambda}(z)\right| r_{1}, \ell, m\right\rangle=\left\langle r_{2}, \ell, m\left|G_{0}(z)\right| r_{1}, \ell, m\right\rangle \\
& +\frac{\left\langle r_{2}, \ell, m\left|G_{0}(z)\right| a, \ell, m\right\rangle\left\langle a, \ell, m\left|G_{0}(z)\right| r_{1}, \ell, m\right\rangle}{\frac{1}{\lambda a^{3}}-\left\langle a, \ell, m\left|G_{0}(z)\right| a, \ell, m\right\rangle}
\end{aligned}
$$

Taking the limit where $\lambda$ goes to infinity, one finally obtains the resolvent partial-wave matrix elements for the hard sphere resolvent:

$$
\begin{gathered}
\left\langle r_{2}, \ell, m|G(z)| r_{1}, \ell, m\right\rangle=\left\langle r_{2}, \ell, m\left|G_{0}(z)\right| r_{1}, \ell, m\right\rangle \\
-\frac{\left\langle r_{2}, \ell, m\left|G_{0}(z)\right| a, \ell, m\right\rangle\left\langle a, \ell, m\left|G_{0}(z)\right| r_{1}, \ell, m\right\rangle}{\left\langle a, \ell, m\left|G_{0}(z)\right| a, \ell, m\right\rangle} .
\end{gathered}
$$

This equation could also be obtained more directly by noting that the right member of equation (4) satisfies the correct differential equations with respect to the variable $r_{1}$ or $r_{2}$ with vanishing boundary conditions at $r_{1}=a$ and at $r_{2}=a$, and correct asymptotic behavior (see equation (5)). An essentially analogous expression for the two-dimensional resolvent for a hard disk is given in [25] with a reference to an unpublished thesis [26]. In [27], the expression for the partial wave hard sphere resolvents (equations (4) and (5)) is given for negative values of the energy (see equation (21) of [27]). In [27], the starting point is different from that of the present paper in two respects. Firstly, it is the resolvent that is expressed as an integral transform of the evolution operator (the first equation of [27]), whereas it is the evolution operator that is expressed as an integral transform of the resolvent in our equation (1). Secondly, imaginary time for the evolution operator is considered in [27] in order to consider 
the Boltzmann operator $\exp (-\beta H)$. An asymptotic series for the resolvent is then inverseLaplace transformed term-by-term in [27] to obtain high-temperature successive quantum corrections for hard spheres second virial coefficients. The interest in the present method is that it gives the partial wave resolvent not only for $\lambda \rightarrow \infty$ (hard sphere), but also for arbitrary $\lambda$ values. The well-known expression for the free resolvent (see, e.g., [28]) is:

$$
\left\langle r_{2}, \ell^{\prime}, m^{\prime}\left|G_{0}\left(E_{+}\right)\right| r_{1}, \ell, m\right\rangle=-\delta_{\ell^{\prime} \ell} \delta_{m^{\prime} m} 2 M p h_{\ell}^{+}\left(p r_{>}\right) j_{\ell}\left(p r_{<}\right)
$$

with

$$
p=(2 M E)^{1 / 2} \text {. }
$$

$r_{>}$is the largest value and $r_{<}$is the smallest value of $r_{2}, r_{1}$. The spherical Bessel functions are defined as in [24]. Specifically, the spherical Bessel functions $j_{\ell}$ and $h_{\ell}^{+}$are related to the usual Bessel function $J_{\ell+1 / 2}$ regular at origin (see e.g [29]) by the equation

$$
\begin{aligned}
& j_{\ell}(z) \equiv\left(\frac{\pi}{2 z}\right)^{1 / 2} J_{\ell+1 / 2}(z) \\
& h_{\ell}^{ \pm}(z) \equiv(-1)^{\ell}\left(\frac{\pi}{2 z}\right)^{1 / 2} J_{-\ell-1 / 2}(z) \pm \mathrm{i} j_{\ell}(z) .
\end{aligned}
$$

It is stressed that $(2 M E)^{1 / 2}$ is defined on the $z$ plane with a cut along the positive real axis. This means that on the first Riemann sheet, the argument of $z$ to be denoted by $\arg 1(z)$ satisfies $0 \leqslant \arg 1(z)<2 \pi$ and $z^{1 / 2}=|z|^{1 / 2} \exp (\mathrm{i} \arg 1(z) / 2)$. Thus $z^{1 / 2}$ should not be confused with $\sqrt{z}$ which is traditionally defined on the $z$ plane cut along the negative real axis, i.e with an $\operatorname{argument} \arg (z)$ which satisfies $-\pi<\arg (z) \leqslant \pi$, so that $\sqrt{z}=|z|^{1 / 2} \exp (\mathrm{i} \arg (z) / 2)$. On the second Riemann sheet of the complex $E$ plane, the argument of $z$ to be denoted by $\arg 2(z)$ satisfies $-2 \pi \leqslant \arg 2(z)<0$ and $z^{1 / 2}=|z|^{1 / 2} \exp (\mathrm{i} \arg 2(z) / 2)$.

One finally obtains

$$
\begin{aligned}
& \left\langle r_{2}, \ell, m\left|G\left(E_{+}\right)\right| r_{1}, \ell, m\right\rangle=\left\langle r_{2}, \ell, m\left|G_{0}\left(E_{+}\right)\right| r_{1}, \ell, m\right\rangle+\left\langle r_{2}, \ell, m\left|G_{n}\left(E_{+}\right)\right| r_{1}, \ell, m\right\rangle \\
& \left\langle r_{2}, \ell, m\left|G_{0}\left(E_{+}\right)\right| r_{1}, \ell, m\right\rangle=-2 M p h_{\ell}^{+}\left(p r_{>}\right) j_{\ell}\left(p r_{<}\right) \\
& \left\langle r_{2}, \ell, m\left|G_{n}\left(E_{+}\right)\right| r_{1}, \ell, m\right\rangle=\frac{2 M p h_{\ell}^{+}\left(p r_{2}\right) h_{\ell}^{+}\left(p r_{1}\right) j_{\ell}(p a)}{h_{\ell}^{+}(p a)},
\end{aligned}
$$

where $G_{n}\left(E_{+}\right)$represents the difference between the full resolvent of the particle in the presence of a hard sphere and the free particle resolvent. From now on, $G_{n}$ will be called the non-free part of the hard sphere resolvent. To avoid any confusion on the vocabulary, we write now several equations as a single index equation:

$$
\left\langle r_{2}, \ell, m\left|U_{k}(t)\right| r_{1}, \ell, m\right\rangle=-\frac{1}{2 \pi \mathrm{i}} \int_{-\infty}^{\infty} \mathrm{d} E \exp \left(-\mathrm{i} \frac{E t}{\hbar}\right)\left\langle r_{2}, \ell, m\left|G_{k}\left(E_{+}\right)\right| r_{1}, \ell, m\right\rangle .
$$

If the index $k$ is absent, the left-hand side is the partial wave hard sphere propagator, and $\left\langle r_{2}, \ell, m\left|G\left(E_{+}\right)\right| r_{1}, \ell, m\right\rangle$ on the right-hand side is the partial wave hard sphere resolvent. If the index $k$ is 0 , the left-hand side is the free partial wave propagator, and $\left\langle r_{2}, \ell, m\left|G_{0}\left(E_{+}\right)\right| r_{1}, \ell, m\right\rangle$ on the right-hand side is the free partial wave resolvent. If the index $k$ is $n$, the left-hand side is the non-free part of the partial wave propagator, and $\left\langle r_{2}, \ell, m\left|G_{n}\left(E_{+}\right)\right| r_{1}, \ell, m\right\rangle$ on the right-hand side is the non-free part of the partial wave resolvent.

Finally, the transition from the partial wave propagator to the propagator is achieved through a series involving Legendre polynomial $P_{\ell}$ :

$$
\left\langle\mathbf{r}_{2}\left|U_{k}(t)\right| \mathbf{r}_{1}\right\rangle=\sum_{\ell=0}^{\infty} \frac{2 \ell+1}{4 \pi} P_{\ell}(\cos (\alpha))\left\langle r_{2}, \ell, m\left|U_{k}(t)\right| r_{1}, \ell, m\right\rangle
$$


with $\alpha$ being the angle between $\mathbf{r}_{2}$ and $\mathbf{r}_{1}$. If the index $k$ is absent, the left-hand side will be called the hard sphere propagator, if the index $k$ is 0 , it will be called the free propagator and if the index $k$ is $n$, it will be called the non-free part of the hard sphere propagator. Alternatively, the partial wave propagator is obtained from the propagator through the relation

$\left\langle r_{2}, \ell, m\left|U_{k}(t)\right| r_{1}, \ell, m\right\rangle=\int \mathrm{d} \Omega_{2} \int \mathrm{d} \Omega_{1} \overline{Y_{\ell}^{m}\left(\Omega_{2}\right)}\left\langle\mathbf{r}_{2}\left|U_{k}(t)\right| \mathbf{r}_{1}\right\rangle Y_{\ell}^{m}\left(\Omega_{1}\right)$

with $Y_{\ell}^{m}$ a spherical harmonic, and $\Omega$ a solid angle.

A well-known exact result (see, e.g., $[1,19,30])$ for the free propagator is

$$
\left\langle\mathbf{r}_{2}\left|U_{0}(t)\right| \mathbf{r}_{1}\right\rangle=\left(\sqrt{\frac{M}{2 \pi \hbar \mathrm{i} t}}\right)^{3} \exp \left(\mathrm{i} \frac{M\left[\mathbf{r}_{2}-\mathbf{r}_{1}\right]^{2}}{2 t \hbar}\right) .
$$

It is worth noting that the modulus of the free propagator is independent of positions and depends only on the length $\sqrt{\hbar t / M}$. From now on, we shall work with units where $\hbar=1$, and we do no longer write the reduced Plank constant in the formulae. From this result, and the relations

$\left\langle\mathbf{r}_{1} \mid r, \ell, m\right\rangle=\frac{\delta\left(r_{1}-r\right)}{r^{2}} Y_{\ell}^{m}\left(\mathbf{r}_{1}\right)$

$\exp \left(\mathrm{i} \beta\left[\mathbf{r}_{2}-\mathbf{r}_{1}\right]^{2}\right)=\exp \left(\mathrm{i} \beta\left(r_{2}^{2}+r_{1}^{2}\right)\right) 4 \pi \sum_{\ell^{\prime}=0}^{\infty} \sum_{m^{\prime}=-\ell^{\prime}}^{\ell^{\prime}}(-\mathrm{i})^{\ell^{\prime}} j_{\ell^{\prime}}\left(2 \beta r_{2} r_{1}\right) Y_{\ell^{\prime}}^{m^{\prime}}\left(\mathbf{r}_{2}\right) \overline{Y_{\ell^{\prime}}^{m^{\prime}}\left(\mathbf{r}_{1}\right)}$

one obtains the partial wave free propagator:

$\left\langle r_{2}, \ell, m\left|U_{0}(t)\right| r_{1}, \ell, m\right\rangle=4 \pi\left(\sqrt{\frac{M}{2 \pi \mathrm{i} t}}\right)^{3} \exp \left(\mathrm{i} \frac{M}{2 t}\left(r_{2}^{2}+r_{1}^{2}\right)\right)(-\mathrm{i})^{\ell} j_{\ell}\left(\frac{M}{t} r_{2} r_{1}\right)$.

\section{An exact analytical result for the $s$ wave hard sphere propagator}

For the s wave, $\ell=0$, the spherical Bessel functions are very simple

$$
\begin{aligned}
& j_{0}(z)=\frac{\sin (z)}{z} \\
& h_{0}^{+}(z)=\frac{\exp (\mathrm{i} z)}{z}
\end{aligned}
$$

and equations (10), (5), (9) give for $r_{2} \geqslant r_{1}$

$$
\begin{aligned}
& \frac{\pi \mathrm{i} r_{2} r_{1}}{M}\left\langle r_{2}, 0,0\left|U_{0}(t)\right| r_{1}, 0,0\right\rangle=\int_{-\infty}^{\infty} \mathrm{d} E \exp (-\mathrm{i} E t) \frac{\exp \left(\mathrm{i} p r_{2}\right) \sin \left(p r_{1}\right)}{p} \\
& -\frac{\pi \mathrm{i} r_{2} r_{1}}{M}\left\langle r_{2}, 0,0\left|U_{n}(t)\right| r_{1}, 0,0\right\rangle=\int_{-\infty}^{\infty} \mathrm{d} E \exp (-\mathrm{i} E t) \frac{\exp \left(\mathrm{i} p\left(r_{2}+r_{1}-a\right)\right) \sin (p a)}{p} .
\end{aligned}
$$

Since the expression for $\left\langle r_{2}, 0,0\left|U_{0}(t)\right| r_{1}, 0,0\right\rangle$ is known from equation (14)

$$
\begin{aligned}
\frac{\pi \mathrm{i} r_{2} r_{1}}{M}\left\langle r_{2}, 0,0\left|U_{0}(t)\right| r_{1}, 0,0\right\rangle & =\frac{\pi \mathrm{i} r_{2} r_{1}}{M} 4 \pi\left(\sqrt{\frac{M}{2 \pi \mathrm{i} t}}\right)^{3} \exp \left(\mathrm{i} \frac{M}{2 t}\left(r_{2}^{2}+r_{1}^{2}\right)\right) j_{0}\left(\frac{M}{t} r_{2} r_{1}\right) \\
& =\sqrt{\frac{2 \pi}{M \mathrm{i} t}} \exp \left(\mathrm{i} \frac{M}{2 t}\left(r_{2}^{2}+r_{1}^{2}\right)\right) \sin \left(\frac{M}{t} r_{2} r_{1}\right) .
\end{aligned}
$$


one obtains the non-free part of the s wave propagator by the substitutions $r_{2} \rightarrow r_{2}+r_{1}-a$ and $r_{1} \rightarrow a$ :

$$
\begin{aligned}
&\left\langle r_{2}, 0,0\right| U_{n}(t)\left|r_{1}, 0,0\right\rangle=-\frac{1}{r_{2} r_{1}} \sqrt{\frac{2 \mathrm{i} M}{\pi t}} \exp \left(\mathrm{i} \frac{M}{2 t}\left(\left(r_{2}+r_{1}-a\right)^{2}+a^{2}\right)\right) \\
& \times \sin \left(\frac{M}{t}\left(r_{2}+r_{1}-a\right) a\right) .
\end{aligned}
$$

\section{An exact expression for the partial wave hard sphere propagator in terms of a Laplace transform}

For the $\ell \neq 0$ partial wave non-free propagators, we resort to numerical computation of the Fourier transform (equations (3), (9)). The integrand is however of doubly oscillating nature on the real positive axis, and numerical integration can fail, or requires too many digits of precision or computation time. This, of course, depends on the numerical values of the parameters $t, a, r_{1}, r_{2}$. For small $t$ values, the difficulty as well as the computation time increases. It is therefore of interest to have another method for comparing numerical results. This other method is based on contour integration and residues theorem. It leads essentially to the numerical computation of a Laplace transform. This numerical method also requires highdigit numbers and a large computation time when $t$ decreases. It is particularly well suitable for large- $t$ values. Anyway, it is useful to have two methods when numerical integration is difficult in order to test accuracy. The expression of the propagator as a Laplace transform is also interesting for study of asymptotic behaviors with respect to different parameters.

The Fourier transform is an integral over the interval $]-\infty,+\infty[$. The integration over the interval ] $-\infty, 0$ [ will be called the left part of the Fourier transform to be denoted by $F_{L}$, the integration over the interval ]0, $\infty$ [ will be called its right part to be denoted by $F_{R}$ :

$$
\begin{aligned}
& F_{L}=-\frac{1}{2 \pi \mathrm{i}} \int_{-\infty}^{0} \mathrm{~d} E \exp (-\mathrm{i} E t)\left\langle r_{2}, \ell, m\left|G_{n}\left(E_{+}\right)\right| r_{1}, \ell, m\right\rangle \\
& F_{R}=-\frac{1}{2 \pi \mathrm{i}} \int_{0}^{+\infty} \mathrm{d} E \exp (-\mathrm{i} E t)\left\langle r_{2}, \ell, m\left|G_{n}\left(E_{+}\right)\right| r_{1}, \ell, m\right\rangle .
\end{aligned}
$$

\subsection{Contour in the complex energy plane for the left part of the Fourier transform}

Figure 1 shows the complex energy plane with its cut along the positive real axis. On the first Riemann sheet, $0 \leqslant \arg (E)<2 \pi$.

The close contour $A O B A$ on the left part of figure 1 is on the first Riemann sheet. It consists of the interval $[-R, 0]$ on the negative real axis, the interval $[0,-\mathrm{i} R]$ on the negative imaginary axis and a quarter circle of radius $R$ starting at the point $-\mathrm{i} R$ and ending at the point $-R$. As the spherical Bessel functions are entire functions, the only singularities of the integrand (9) are possibly at the zeros of $h_{\ell}^{+}\left((2 M E)^{1 / 2} a\right)$. The $\ell$ zeros of $h_{\ell}^{+}(z)$ in the $z$ plane have negative imaginary part (see [29]). For $\ell=1$, one zero at $z=-\mathrm{i}$. For $\ell=2,3, \ldots, 10$, they are shown in figure 2 .

With the change of variable given by equation (6), the close contour $A O B A$ in the $E$ plane becomes the close contour $a O b a$ in the $p$ plane (see figure 3 ).

It consists of the interval $[\mathrm{i} \sqrt{2 M R}, 0]$ on the positive imaginary axis, the interval $\left[0, \sqrt{2 M R} \exp \left(\mathrm{i} \frac{3 \pi}{4}\right)\right]$ on the second bisector and part of a circle of radius $\sqrt{2 M R}$ starting at the point $\sqrt{2 M R} \exp \left(\mathrm{i} \frac{3 \pi}{4}\right)$ and ending at the point $\mathrm{i} \sqrt{2 M R}$. The contour $a O b a$ does not contain zero of $h_{\ell}^{+}$and the integral along the close contour $A O B A$ is zero. As the 


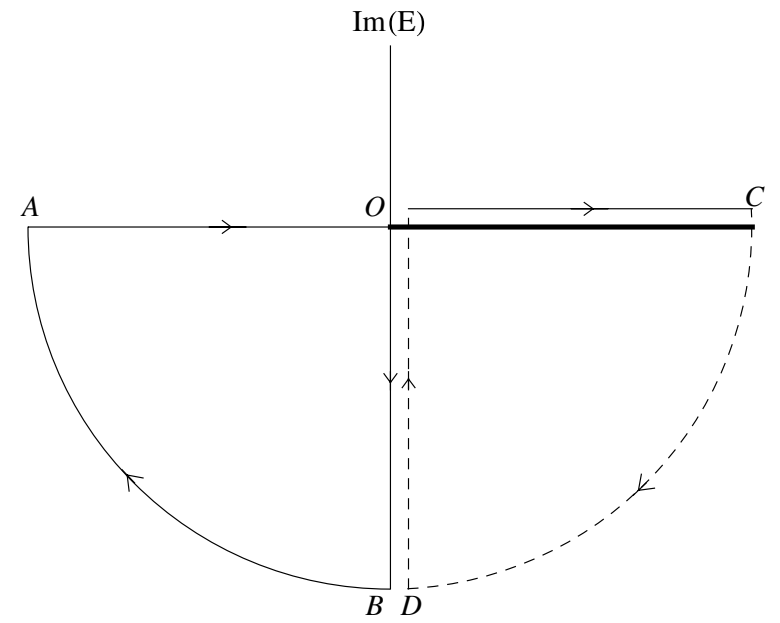

Figure 1. Contours in the cut energy plane $E$. The dashed line curve lies on the second Riemann sheet.

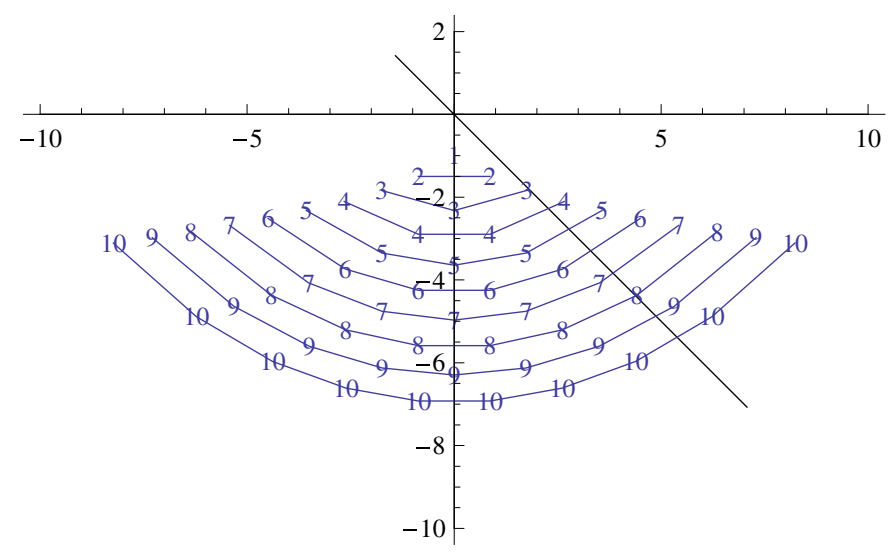

Figure 2. Zeros of the spherical Bessel function $h_{\ell}^{+}(z)$ in the $z$ plane. For example the nine 9 on the figure are centered on the nine zeros of $h_{9}^{+}(z)$.

contribution of the integration along the circular part goes to zero when $R \rightarrow+\infty$, the left part of the Fourier transform is equal to the integral along the negative imaginary axis ]-i $\infty, 0\left[\right.$ on the first Riemann sheet of the $E$ plane, where $E=|E| \exp \left(\mathrm{i} \frac{3 \pi}{2}\right)$, and therefore $p=\sqrt{2 M|E|} \exp \left(\mathrm{i} \frac{3 \pi}{4}\right)=-\sqrt{2 M|E|} \exp \left(-\mathrm{i} \frac{\pi}{4}\right)$. From the symmetry properties of the spherical Bessel function

$$
j_{\ell}(-z)=(-1)^{\ell} j_{\ell}(z) \quad h_{\ell}^{+}(-z)=(-1)^{\ell+1} h_{\ell}^{-}(z) .
$$

With the change of variable $E=-\mathrm{i} u$, one obtains finally an integral on the real positive axis which is a Laplace transform:

$$
F_{L}=\frac{M}{\pi} \int_{0}^{\infty} \mathrm{d} u \exp (-u t) z(u) j_{\ell}(z(u) a)\left\{-\frac{h_{\ell}^{-}\left(z(u) r_{2}\right) h_{\ell}^{-}\left(z(u) r_{1}\right)}{h_{\ell}^{-}(z(u) a)}\right\}
$$




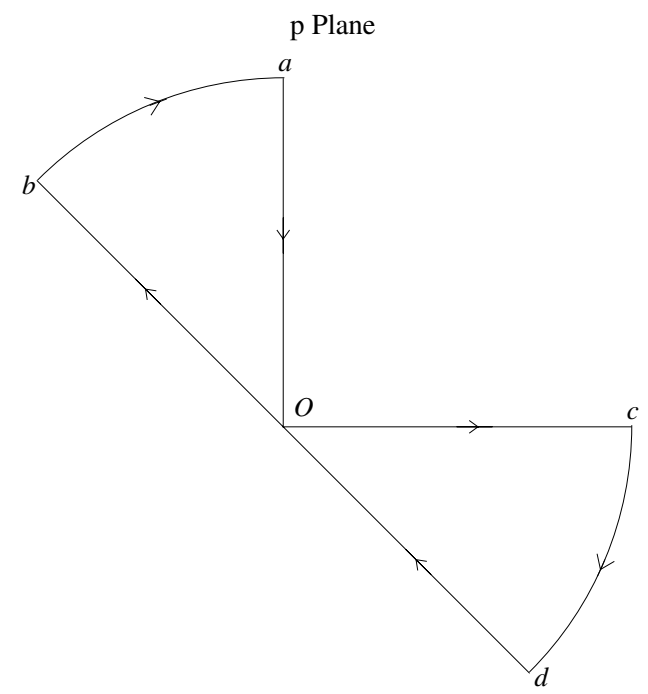

Figure 3. Contours in the $p$ complex plane, corresponding to the contours of figure 1.

with

$$
z(u)=\sqrt{2 M u} \exp \left(-\mathrm{i} \frac{\pi}{4}\right)
$$

\subsection{Contour in the complex energy plane for the right part of the Fourier transform}

In order to have a close contour involving the integral on the real positive axis (first sheet of the $E$ plane) with a vanishing contribution of a quarter circle, the imaginary part on the quarter circle must be negative. Since the $E$ plane is cut along the real positive axis, the integrand must be analytically continued into the second Riemann sheet. The closed contour $O C D O$ in figure 1 is the following: $O C$ is the interval $[0, R]$ on the real positive axis (first sheet of the $E$ plane), $C B$ is a quarter circle of radius $R$ in the second Riemann sheet and $D O$ is the purely imaginary interval $[-\mathrm{i} R, 0]$ in the second Riemann sheet. The analytical continuation of the integrand into the second Riemann sheet is simply achieved by the equation $E=|E| \exp (\mathrm{i} \alpha)$ with now $-\frac{\pi}{2} \leqslant \alpha \leqslant 0$.

With the change of variable given by equation (6), the close contour $O C D O$ in the $E$ plane becomes the close contour $O c d O$ in the $p$ plane (see figure 3). It is seen in figures 2 and 3 that for $\ell \geqslant 4$, the integrand has poles inside the contour with respect to the variable $p$. The number of poles increases slowly with $\ell$ : one pole for $\ell$ from 4 to 7 , two poles for $\ell$ from 8 to 12 , etc. The right part of the Fourier transform is finally equal to minus the integral along the negative imaginary axis ]-i $\infty, 0$ [ on the second Riemann sheet of the $E$ plane, where $E=|E| \exp \left(-\mathrm{i} \frac{\pi}{2}\right)$, and therefore $p=\sqrt{2 M|E|} \exp \left(-\mathrm{i} \frac{\pi}{4}\right)$, minus the contribution of the poles. With the change of variable $E=-\mathrm{i} u$, one finally obtains an integral on the real positive axis which is a Laplace transform:

$F_{R}=\frac{M}{\pi} \int_{0}^{\infty} \mathrm{d} u \exp (-u t) z(u) j_{\ell}(z(u) a) \frac{h_{\ell}^{+}\left(z(u) r_{2}\right) h_{\ell}^{+}\left(z(u) r_{1}\right)}{h_{\ell}^{+}(z(u) a)}+\sum_{p_{0}} \operatorname{residues}(f(p))$ 
with $z(u)$ again defined by equation (16), and

$$
f(p)=2 \exp \left(-\mathrm{i} \frac{p^{2}}{2 M} t\right) \frac{p^{2} h_{\ell}^{+}\left(p r_{2}\right) j_{\ell}(p a) h_{\ell}^{+}\left(p r_{1}\right)}{h_{\ell}^{+}(p a)} .
$$

The summation over $p_{0}$ is over all zeros of $h_{\ell}^{+}(p a)$ with respect to the variable $p$ between the real positive axis and the second bisector (see figure 2). For $\ell<4$, this summation over $p_{0}$ is empty and therefore yields 0 .

\subsection{Final results}

Let us summarize the results. The partial wave hard sphere propagator for a sphere of radius $a$ is given by

$\left\langle r_{2}, \ell, m|U(t)| r_{1}, \ell, m\right\rangle=\left\langle r_{2}, \ell, m\left|U_{0}(t)\right| r_{1}, \ell, m\right\rangle+\left\langle r_{2}, \ell, m\left|U_{n}(t)\right| r_{1}, \ell, m\right\rangle$

where the free part $\left\langle r_{2}, \ell, m\left|U_{0}(t)\right| r_{1}, \ell, m\right\rangle$ is given by equation (14) and where the non-free part of the partial wave propagator $\left\langle r_{2}, \ell, m\left|U_{n}(t)\right| r_{1}, \ell, m\right\rangle$ is given by the Fourier transform:

$\left\langle r_{2}, \ell, m\left|U_{n}(t)\right| r_{1}, \ell, m\right\rangle=-\frac{M}{\pi \mathrm{i}} \int_{-\infty}^{\infty} \mathrm{d} E \exp (-\mathrm{i} E t) \frac{p h_{\ell}^{+}\left(p r_{2}\right) h_{\ell}^{+}\left(p r_{1}\right) j_{\ell}(p a)}{h_{\ell}^{+}(p a)}$

with $p$ defined by equation (6). Another expression for the non-free part of the partial wave propagator is given by a Laplace transform (with residues contributions if $\ell \geqslant 4$ ):

$$
\begin{aligned}
& \left\langle r_{2}, \ell, m\left|U_{n}(t)\right| r_{1}, \ell, m\right\rangle=\frac{M}{\pi}\left\{\begin{array}{l}
\int_{0}^{\infty} \mathrm{d} u \exp (-u t) z(u) j_{\ell}(z(u) a) \\
{\left[\frac{h_{\ell}^{+}\left(z(u) r_{2}\right) h_{\ell}^{+}\left(z(u) r_{1}\right)}{h_{\ell}^{+}(z(u) a)}-\frac{h_{\ell}^{-}\left(z(u) r_{2}\right) h_{\ell}^{-}\left(z(u) r_{1}\right)}{h_{\ell}^{-}(z(u) a)}\right]}
\end{array}\right\} \\
& +\sum_{p_{0}} \text { residues }(f(p))
\end{aligned}
$$

with $z(u)$ defined by equation (16) and $f(p)$ defined by equation (17). The summation over $p_{0}$ is over all zeros of $h_{\ell}^{+}(p a)$ whose arguments are in the interval $]-\pi / 4,0[$.

For $\mathrm{s}$ wave $(\ell=0)$, the integration has been done analytically and yields the result given by equation (15).

\section{Numerical results}

\subsection{Comparison with a quantal approximation}

Several attempts to approximate analytical expressions for the hard sphere propagator has been made in the past $[4,5]$. These approximations have been called image approximations. More recently, the $\mathrm{CB}$ [7] approximation has superseded image approximations. The $\mathrm{CB}$ propagator, noted $\left\langle\mathbf{r}_{2}\left|U_{C B}(t)\right| \mathbf{r}_{1}\right\rangle$ is [7]:

$$
\left\langle\mathbf{r}_{2}\left|U_{C B}(t)\right| \mathbf{r}_{1}\right\rangle=\left(\frac{M}{\mathrm{i} 2 \pi t}\right)^{3 / 2}\left[\exp \left(\mathrm{i} \frac{M\left(\mathbf{r}_{2}-\mathbf{r}_{1}\right)^{2}}{2 t}\right)-\frac{a(u-a)}{r_{2} r_{1}} \exp \left(\mathrm{i} \frac{M D}{2 t}\right)\right]
$$

with

$$
\begin{aligned}
& u=r_{2}+r_{1} \\
& D=u^{2}-2 a u+2 a^{2}-2 a(u-a) \cos (\alpha) .
\end{aligned}
$$

The first term involving $\exp \left(\mathrm{i} \frac{M\left(\mathbf{r}_{2}-\mathbf{r}_{1}\right)^{2}}{2 t}\right)$ corresponds to the free propagator, and the non-free part of the CB propagator, to be denoted by $\left\langle\mathbf{r}_{2}\left|U_{C B n}(t)\right| \mathbf{r}_{1}\right\rangle$ is thus

$$
\left\langle\mathbf{r}_{2}\left|U_{C B n}(t)\right| \mathbf{r}_{1}\right\rangle=-\left(\frac{M}{\mathrm{i} 2 \pi t}\right)^{3 / 2} \frac{a(u-a)}{r_{2} r_{1}} \exp \left(\mathrm{i} \frac{M D}{2 t}\right) .
$$


Table 1. First line: $\ell$, exact non-free part of the partial wave propagator $\left\langle r_{2}, \ell, m\left|U_{n}(t)\right| r_{1}, \ell, m\right\rangle$ (see equations (19), (20)), non-free part of the CB propagator $\left\langle r_{2}, \ell, m\left|U_{C B n}(t)\right| r_{1}, \ell, m\right\rangle$ (see equation (22)), for $M=1, t=1, a=1, r_{1}=2, r_{2}=3$.

\begin{tabular}{lcl}
\hline$\ell$ & $\left\langle 3, \ell, m\left|U_{n}(1)\right| 2, \ell, m\right\rangle$ & $\left\langle 3, \ell, m\left|U_{C B n}(1)\right| 2, \ell, m\right\rangle$ \\
\hline 0 & $0.0997-0.0140 \mathrm{i}$ & $0.0997-0.0140 \mathrm{i}$ \\
1 & $0.0122+0.0565 \mathrm{i}$ & $0.0086+0.0612 \mathrm{i}$ \\
2 & $0.1216-0.0560 \mathrm{i}$ & $0.146-0.020 \mathrm{i}$ \\
3 & $-0.0746-0.0764 \mathrm{i}$ & $-0.0169-0.1208 \mathrm{i}$ \\
4 & $-0.0165+0.0536 \mathrm{i}$ & $-0.0658+0.0092 \mathrm{i}$ \\
5 & $0.0206-0.0085 \mathrm{i}$ & $0.0038+0.0273 \mathrm{i}$ \\
6 & $-0.00676-0.00168 \mathrm{i}$ & $0.0162-0.0023 \mathrm{i}$ \\
7 & $0.00140+0.00095 \mathrm{i}$ & $-0.00037-0.00263 \mathrm{i}$ \\
8 & $-0.000239-0.000174 \mathrm{i}$ & $-0.000652+0.000091 \mathrm{i}$ \\
9 & $0.0000307+0.0000116 \mathrm{i}$ & $0.000020+0.000143 \mathrm{i}$ \\
\hline
\end{tabular}

One deduces from equation (12) the non-free part of the partial wave CB propagator:

$$
\begin{gathered}
\left\langle r_{2}, \ell, m\left|U_{C B n}(t)\right| r_{1}, \ell, m\right\rangle=-4 \pi\left(\sqrt{\frac{M}{2 \pi i t}}\right)^{3} \frac{a(u-a)}{r_{2} r_{1}} \exp \left(i \frac{M\left(u^{2}-2 a u+2 a^{2}\right)}{2 t}\right) \\
\times(-i)^{\ell} j_{\ell}\left(\frac{M a(u-a)}{t}\right) .
\end{gathered}
$$

Comparison with equation (15) shows that the $\mathrm{CB}$ propagator gives the exact result for the s wave $\ell=0$. However, the CB partial wave propagator rapidly becomes inaccurate when $\ell$ increases, as shown in table 1 . The modulus of the CB partial wave propagator does not decrease sufficiently rapidly for large $\ell$ values. For example, for $\ell=25$, the exact result is $(-3.02+1.98 \mathrm{i}) \times 10^{-30}$ whereas the CB result is $(2.4+17.1 \mathrm{i}) \times 10^{-20}$.

It is of interest to see how the non-free part of the CB partial wave propagator can be obtained from the exact result (equation (19)). One first replaces all $h_{\ell}^{+}$functions of the integrand by their asymptotic form for large argument: $h_{\ell}^{+}(z) \simeq \exp \left(\mathrm{i} z-\ell \frac{\pi}{2}\right) / z$. This gives $\frac{a}{p r_{2} r_{1}} \exp \left(\mathrm{i}\left(p\left(r_{2}+r_{1}-a\right)-\ell \frac{\pi}{2}\right)\right)$. Now one considers the limit form of all $h_{\ell}^{+}$ functions of the integrand for small argument: $h_{\ell}^{+}(z) \simeq z^{-\ell-1}(2 \ell+1) ! ! /(2 \ell+1)$. This gives $p^{-\ell-1}\left(a /\left(r_{1} r_{2}\right)\right)^{\ell+1}(2 \ell+1) ! ! /(2 \ell+1)$. Then the replacement of all $h_{\ell}^{+}$functions of the integrand by $\frac{a}{r_{2} r_{1}}\left(r_{2}+r_{1}-a\right) h_{\ell}^{+}\left(p\left(r_{2}+r_{1}-a\right)\right)$ ensures the correct asymptotic behavior for large argument and a correct behavior with respect to $p$, namely proportional to $p^{-\ell-1}$ in the limit of small $p$ values. The Fourier transform then can be evaluated analytically and one obtains equation (22). Since $h_{0}^{+}(z)$ coincides with its asymptotic form, one understands why the Cao-Berne partial wave propagator is exact for $\ell=0$. One understands also why the disagreement increases with $\ell$ since the asymptotic behavior of $h_{\ell}^{+}(z)$ is attained for increasing values of $z$ when $\ell$ increases.

Summation over partial waves (see equation (11)) gives the propagator as a function of the angle $\alpha$ between the two vectors $\mathbf{r}_{2}, \mathbf{r}_{1}$ (see figure 4). Specifically, the hard sphere propagator has been numerically calculated by adding its free part computed according to equation (13), and its non-free part computed by summing the partial wave propagators (equation (11) with index $k$ equal to $n$ ). The critical values $\alpha_{c}$ for which the vector $\mathbf{r}_{2}-\mathbf{r}_{1}$ is tangent to the hard sphere of radius $a$ are given by the geometrical relation

$$
\alpha_{c}=\arccos \left(\frac{a}{r_{1}}\right)+\arccos \left(\frac{a}{r_{2}}\right) .
$$




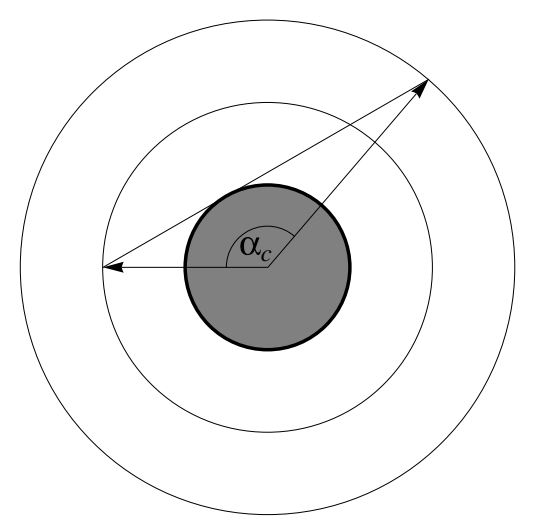

Figure 4. The origin of the two vectors $\mathbf{r}_{1}, \mathbf{r}_{2}$ is the center of a hard sphere of radius $a=1 . \alpha$ is the angle between $\mathbf{r}_{1}, \mathbf{r}_{2}$. The norms of the two vectors are constant, $r_{1}=2, r_{2}=3$. The extremal values of $\left|\mathbf{r}_{2}-\mathbf{r}_{1}\right|$ are 1 (parallel case) and 5 (antiparallel case). When $\mathbf{r}_{2}-\mathbf{r}_{1}$ becomes tangent to the hard sphere of radius $a$, the angle $\alpha$ has the critical value $\alpha_{c}$ above which no classical trajectory exists.
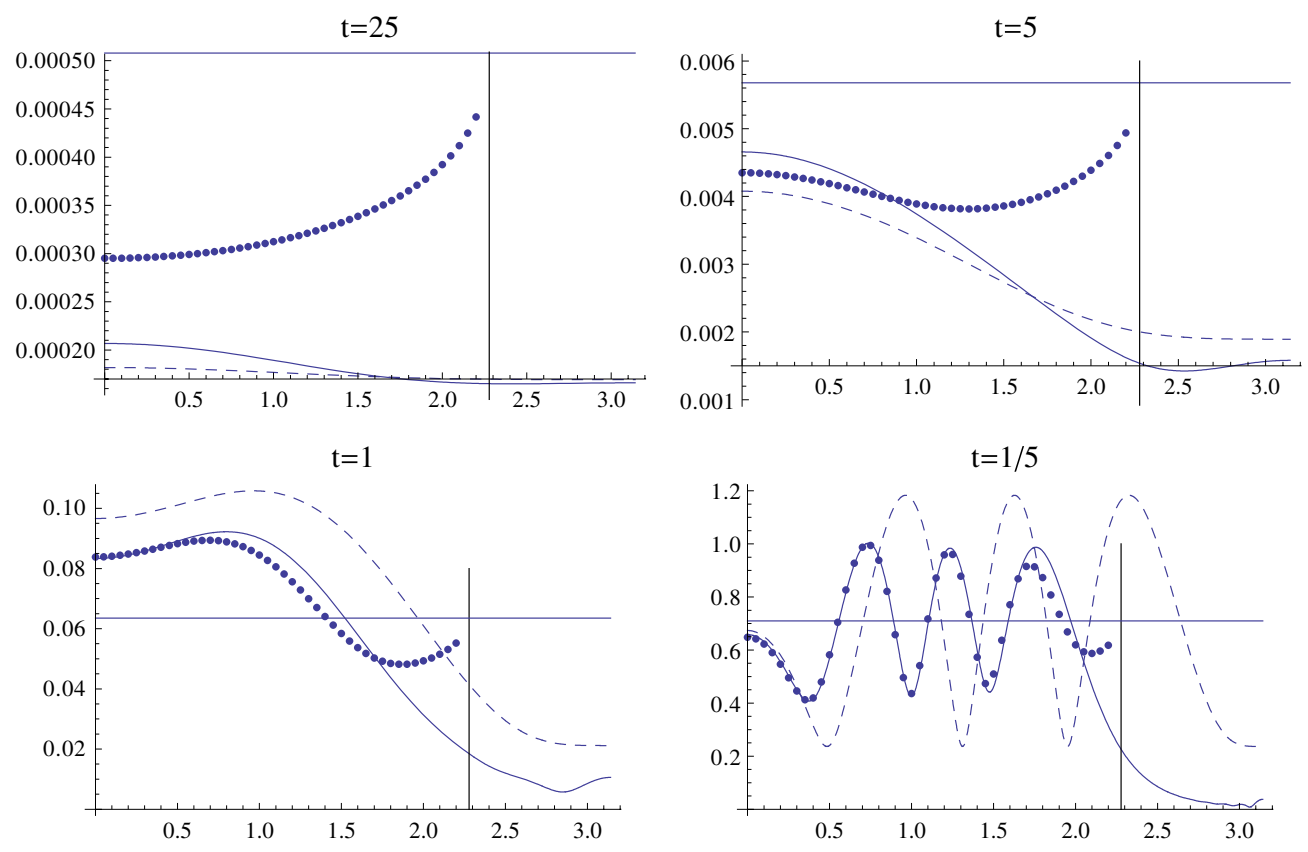

Figure 5. Modulus of the hard sphere quantum propagator as a function of the angle $\alpha$ between the two vectors $\mathbf{r}_{1}, \mathbf{r}_{2}$ for the geometrical configurations of figure 4 ( $a=1, r_{1}=2, r_{2}=3$ ), for $M=1$, and $t=25,5,1,1 / 5$. Full curve: exact result. Dashed curve: Cao-Berne approximation (see equation (21)). Points: Van Vleck-Gutzwiller approximation (see equation (24)). The vertical line indicates the value of the critical angle $\alpha_{c}$. The horizontal lines indicate the values of the modulus of the free propagator (see equation (13)).

We call the domain $\alpha<\alpha_{c}$ the classical domain, and the domain $\alpha>\alpha_{c}$ the classically forbidden domain.

Exact numerical results for the modulus of the hard sphere propagator are compared with the $\mathrm{CB}$ approximation in figures 5 and 6. In these figures, the $\mathrm{CB}$ approximation reproduces 


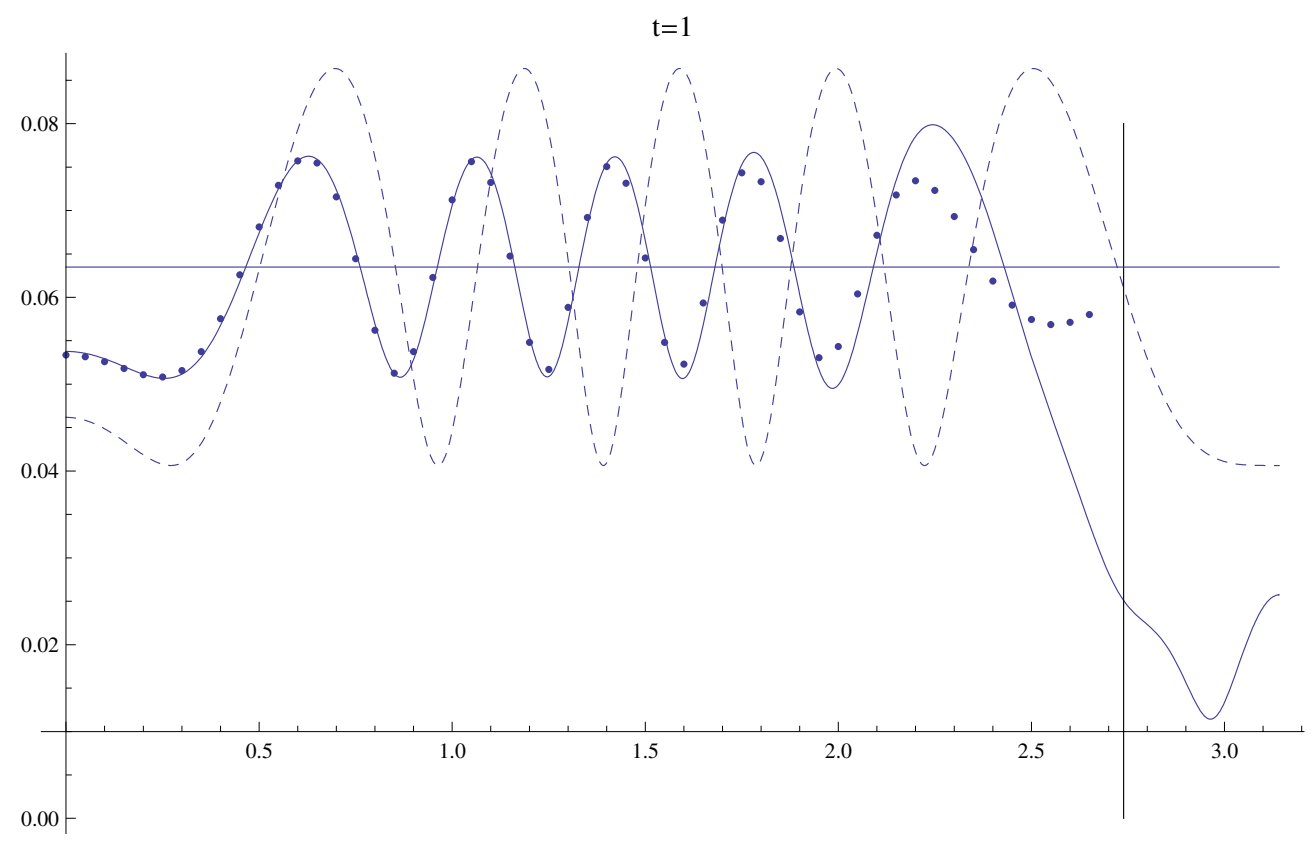

Figure 6. Modulus of the hard sphere quantum propagator as a function of the angle $\alpha$ between the two vectors $\mathbf{r}_{1}, \mathbf{r}_{2}$ for $a=1, r_{1}=r_{2}=5, M=1, t=1$. Full curve: exact result. Dashed curve: Cao-Berne approximation (See equation (21)). Points: Van Vleck-Gutzwiller approximation (See equation (24)). The vertical line indicates the value of the critical angle $\alpha_{c}$. The horizontal lines indicate the values of the modulus of the free propagator (see equation (13)).

the main qualitative behavior of the exact results. In the classical domain, the oscillations have a very large amplitude and the phase may be incorrect, although the number of oscillations is correct for the examples considered. The exact results exhibit a significant decrease before coming into the classically forbidden domain. This decrease is physically quite natural. The CB approximation does not always decrease sufficiently, and, for example, an unphysical maximum just after $\alpha_{c}$ for $t=1 / 5$ appears. To summarize, the CB approximation has been found globally qualitatively correct but quantitatively inaccurate.

An interesting phenomenon exhibited by the exact results is a local maximum for $\alpha=\pi$. This effect is particularly visible in figure 6. This counter intuitive effect from a classical view point is a purely quantal reminiscent of a similar effect in optics: central bright spot (called Maraldi or Poisson or Arago spot) at the centre of the geometrical shadow in Fresnel diffraction by a circular obstacle [31, 32].

\subsection{Classical arguments and limit cases}

How rapidly does the summation over partial wave converge (see equation (11))? An estimation for the maximum value $\ell_{M}$ to be used for truncating the series (11) is obtained by looking at a majorant for the classical angular momentum compatible with $M, a, r_{1}, r_{2}, t$ values. For fixed values of the velocity $v$, the angular momentum is maximum at maximal impact parameter $a$, when the trajectory is tangent to the hard sphere. The modulus of the angular momentum in this configuration is the product $a M v$. For a given time $t$, the velocity is maximum for the maximum distance along trajectories compatible with $r_{1}$ and $r_{2}$. The 
trajectory distance between $\mathbf{r}$ and the hard sphere is maximum for the tangential trajectory, and is then $\sqrt{r^{2}-a^{2}}$. A classical majorant for angular momentum is then:

$$
\ell_{c}=a M \frac{\sqrt{r_{1}^{2}-a^{2}}+\sqrt{r_{2}^{2}-a^{2}}}{t} \text {. }
$$

One therefore expects that the contribution to the sum of the series (11) should be negligible for $\ell$ greater than about

$$
\ell_{M}=\alpha \frac{a M\left(r_{1}+r_{2}\right)}{t}+\beta .
$$

The choice for the values of the coefficients $\alpha, \beta$ depends on the precision required. The choice $\alpha=3 / 2$ and $\beta=5$ has been found largely sufficient for the precision of drawing figures 5 and 6 . The reason for the addition of the number $\beta$ is that for small quantum numbers, classical argument may fail. For the cases considered in figure $5, \ell_{M}$ is respectively equal to $5.3,6.5,12.5$ and 42.5 , for $t$ respectively equal to $25,5,1$ and $1 / 5$. The values of $\ell$ above which the visual appearance of the graph does not change are 2, 2, 7, 30, for $t$ respectively equal to $25,5,1$ and $1 / 5$. The calculations have been made up to $\ell$ equal to $7,15,25$ and 50 for $t$ respectively equal to $25,5,1$ and $1 / 5$ and it has been verified that the decrease is very rapid above $\ell_{M}$. For the case considered in figure $6, \ell_{M}$ is equal to 20 and it has been verified that the visual appearance of the graph does not change as long as the truncation value is equal to or above 13. If one requires increasing the relative accuracy, the majorant must of course also be increased.

Physically, an important result is that in the limit $a \rightarrow 0$ or $t \rightarrow \infty$ or $M \rightarrow 0$, only the $\mathrm{s}$ and $\mathrm{p}$ waves contribute significantly. (Of course the limit $M \rightarrow 0$ is not a physical one.) What about the opposite cases, the short time limit? It will be seen in the next section that the classical result of Van Vleck-Gutzwiller provides an excellent approximation in the classical domain.

\subsection{Comparison with a classical approximation}

In the classical region $\alpha \leqslant \alpha_{c}$ (see equation (23)), or otherwise stated, for $\mathbf{r}_{2}$ outside the geometric shadow of the hard sphere of radius $a$, the VG approximation for the hard sphere quantum propagator, to be denoted by $\left\langle\mathbf{r}_{2}\left|U_{V G}(t)\right| \mathbf{r}_{1}\right\rangle$ is $[18,19]$

$$
\left\langle\mathbf{r}_{2}\left|U_{V G}(t)\right| \mathbf{r}_{1}\right\rangle=\left\langle\mathbf{r}_{2}\left|U_{0}(t)\right| \mathbf{r}_{1}\right\rangle-\frac{1}{(2 \pi \mathrm{i})^{3 / 2}} \sqrt{\operatorname{det}\left|-\frac{\partial^{2} S}{\partial \mathbf{r}_{2} \partial \mathbf{r}_{1}}\right|} \operatorname{exp(iS)}
$$

In equation (24), $\left\langle\mathbf{r}_{2}\left|U_{0}(t)\right| \mathbf{r}_{1}\right\rangle$ is the free propagator (see equation (13)). $S$ is the classical action, the integral over time of the Lagrangian $L: \int_{\mathbf{r}_{1}}^{\mathbf{r}_{2}} L \mathrm{~d} t=\int_{\mathbf{r}_{1}}^{\mathbf{r}_{2}} \frac{p^{2}}{2 M} \mathrm{~d} t$ for the real classical trajectory starting at time 0 at the point $\mathbf{r}_{1}$ and ending at time $t$ at the point $\mathbf{r}_{2}$ after one collision with the hard sphere of radius $a$. (The present action $S$ is called the Hamilton's principal function in $[19,25]$, where it is noted $R$. In these references, the name action and the letter $S$ refer to another function.) The minus sign on the right-hand side of equation (24) originates from the reflection at the point of collision with the hard sphere. The action $S$ is thus a function of the variables $M, t, a, \mathbf{r}_{2}, \mathbf{r}_{1}$. Finally $\frac{\partial^{2} S}{\partial \mathbf{r}_{2} \partial \mathbf{r}_{1}}$ denotes the matrix of order 3 whose $i j$ element is the second derivative $\frac{\partial^{2} S}{\partial\left(x_{2}\right)_{i} \partial\left(x_{1}\right)_{j}}$ of the action with respect to the coordinates of the end points $\mathbf{r}_{2}, \mathbf{r}_{1}$ of the classical trajectories. As emphasized in [19], the burden of computing explicitly $\left\langle\mathbf{r}_{2}\left|U_{V G}(t)\right| \mathbf{r}_{1}\right\rangle$ lies completely within classical mechanics, and the VG approximation can appropriately also be called the classical propagator. Specifically

$$
S=\frac{M}{2 t} l^{2}
$$


with $l$ the length of the classical trajectory connecting the points $\mathbf{r}_{1}, \mathbf{r}_{2}$ with one collision with the hard sphere. In the applications to be discussed now, the length $l$ has been determined numerically by minimization, and the second derivative of $S$ has been determined numerically according to the relation [29]

$\frac{\partial^{2} f}{\partial x \partial y}(x, y) \simeq \frac{f(x+b, y+b)-f(x-b, y+b)-f(x+b, y-b)+f(x-b, y-b)}{4 b^{2}}$

The accuracy has been tested by changing the values of $b$. For example, for $b=1 / 100$ and $b=1 / 10$, the relative change of all points in figures 5 and 6 (to be defined as the ratio between the result with $b=1 / 10$ minus the result with $b=1 / 100$ and the result with $b=1 / 100$ ) is less than $5 \times 10^{-4}$.

It is seen in figure 5 that the accuracy of the VG approximation increases when $t$ decreases. This accuracy is excellent for $t=1 / 5$, except when approaching the classically forbidden region $\left(\alpha \simeq \alpha_{c}\right)$. This also holds for figure 6 . This behavior with respect to time was expected since the classical action increases when $t$ decreases or when $l$ increases (see equation (25)). The action $S$ increases with $\alpha$. For figure 5, the minimum values of $S$, for $\alpha=0$, are $0.18 \hbar, 0.9 \hbar, 4.5 \hbar, 22.5 \hbar$ for $t=25,5,1,1 / 5$, respectively. For figure 6 , the minimum value of $S$, for $\alpha=0$, is $32 \hbar$. Path integral formulation of quantum mechanics [33, 1, 34] tells us that for a very large value of the classical action, the sum over all paths (classical and non-classical) is dominated by the classical path due to the stationary value of the action for this path. It is therefore natural that the accuracy of the VG propagator increases when $S$ increases (at a fixed value of $\alpha$ ). What is remarkable is the degree of accuracy for such low values of the action $S$, only a few units of $\hbar$. This is however not quite a surprise since the exact value of the free propagator (see equation (13)) coincides with the free VG propagator, and the real classical trajectory with one collision with the hard sphere is nothing but two free trajectories (one before the collision, one after).

Only the moduli of propagators are reported in figures 5 and 6, but when the modulus of the approximate propagators agrees with the modulus of the exact result, the real and imaginary parts also agree.

\section{Conclusion}

By an explicit determination of the matrix elements of the hard sphere resolvent (see equation (4)), an exact expression for the partial wave hard sphere quantum propagator in a three-dimensional space has been given in terms of a Fourier transform of an expression involving spherical Bessel functions. For the s wave case, the Fourier transform has been calculated analytically. This Fourier transform has also been evaluated for the general case by contour integration in terms of a Laplace transform and residues contribution. This Laplace transform is particularly suitable for numerical computation for the large time value $t$ (small values of the classical action $S$ (see equation (25)). For small $t$ values (more generally for large values of the classical action $S$ in units of $\hbar$ ), numerical calculations by both methods (Fourier and Laplace) are difficult, and require a large number of digits for precision and a large computation time.

The calculation of the hard sphere quantum propagator by summation over partial wave contribution has shown an excellent accuracy of the Van Vleck-Gutzwiller approximation in the classical domain $\alpha<\alpha_{c}$ for $\alpha$ not in the vicinity of $\alpha_{c}$, and when the classical action $S$ (see equation (25)) is greater than only a few units of $\hbar$. In the classically forbidden domain $\left(\alpha>\alpha_{c}\right)$, the exact result rapidly decreases as expected, but exhibits a small local maximum for $\alpha=\pi$, i.e. for the point $\mathbf{r}_{2}$ on the other side of the sphere, so that the three points $\mathbf{r}_{1}$, 
the center of the sphere and $\mathbf{r}_{2}$ are aligned. This effect is reminiscent of Arago bright spot in Fresnel diffraction [31,32].

\section{Acknowledgments}

The author thanks the referees for drawing his attention to reference [27].

\section{References}

[1] Feynman R P and Hibbs A R 1965 Quantum Mechanics and Path Integrals (New York: McGraw-Hill)

[2] Beauregard L A 1966 Am. J. Phys. 34324

[3] Bjorken J D and Drell S D 1966 Relativistische Quantenmechanik Band 98 (Mannheim, Bibliographisches Institut Hochschultaschenbücher)

[4] Uhlenbeck G E and Beth E 1936 Phys. Fenn. 3729

[5] Barker J 1979 J. Chem. Phys 702914

[6] Jacucci G and Omerti E 1983 J. Chem. Phys. 79305

[7] Cao J and Berne J 1992 J. Chem. Phys. 972382

[8] Grüter P, Ceperley D and Laloë F 1997 Phys. Rev. Lett. 793549

[9] Sesé L M and Ledesma R 1995 J. Chem. Phys. 1023776

[10] Sesé L M and Ledesma R 1997 J. Chem. Phys. 1061134

[11] Sesé L M 1998 J. Chem. Phys. 1089086

[12] Sesé L M 2001 J. Chem. Phys. 1141732

[13] Bailey L E and Sesé L M 2001 J. Chem. Phys. 1156557

[14] Sesé L M 2002 J. Chem. Phys. 1168492

[15] Nho K and Landau D P 2004 Phys. Rev. A 70053614

[16] Nho K and Landau D P 2005 Phys. Rev. A 70023615

[17] Van Vleck J H 1928 Proc. Natl Acad. Sci. USA 14178

[18] Gutzwiller M C 1967 J. Math. Phys. 81979

[19] Gutzwiller M C 1990 Chaos in Classical and Quantum Mechanics (New York: Springer)

[20] Goussev A and Dorfman J R Phys. Rev. E 71026225

[21] Cohen-Tannoudji C, Dupont-Roc J and Grynberg G 1988 Processus D'interaction Entre Photons et Atomes (Paris: InterEditions/Editions du CNRS)

[22] de Prunelé E 1997 J. Phys. A: Math. Gen. 307831

[23] Morse P M and Feshbach H 1953 Methods of Theoretical Physics, Part II (New York: McGraw-Hill) p 2483

[24] Messiah A 1962 Mécanique quantique-1 (Paris: Dunod)

[25] Brack M and Bhaduri R K 1997 Semiclassical Physics (Reading, MA: Addison-Wesley) pp 342 and 357

[26] Snaith N C 1996 B.Sc Thesis McMaster University, unpublished

[27] Hill R N 1968 J. Math. Phys. 91534

[28] Taylor J R 1972 Scattering Theory (New York: Wiley)

[29] Abramowitz M and Stegun I A 1965 Handbook of Mathematical Functions (New York: Dover)

[30] Durand E 1970 Mécanique Quantique Tome 1 (Paris: Masson et $\mathrm{C}^{\mathrm{ie}}$ )

[31] Ditchburn R W 1976 Light (London: Academic)

[32] Born M and Wolf E 1999 Principles of Optics (Cambridge: Cambridge University Press) p 417

[33] Feynman R P 1948 Rev. Mod. Phys. 20367

[34] Zinn-Justin J 2003 Intégrale de Chemin en Mé canique Quantique: Introduction (Les Ulis, Paris: EDP Sciences CNRS Editions) 